\title{
GLOBAL ATTRACTIVITY OF POSITIVE PERIODIC SOLUTIONS FOR AN IMPULSIVE DELAY PERIODIC "FOOD LIMITED" POPULATION MODEL
}

\author{
JIAN SONG
}

Received 14 February 2006; Accepted 16 May 2006

We will consider the following nonlinear impulsive delay differential equation $N^{\prime}(t)=$ $r(t) N(t)((K(t)-N(t-m w)) /(K(t)+\lambda(t) N(t-m w)))$, a.e. $t>0, t \neq t_{k}, N\left(t_{k}^{+}\right)=(1+$ $\left.b_{k}\right) N\left(t_{k}\right), K=1,2, \ldots$, where $m$ is a positive integer, $r(t), K(t), \lambda(t)$ are positive periodic functions of periodic $\omega$. In the nondelay case $(m=0)$, we show that the above equation has a unique positive periodic solution $N^{*}(t)$ which is globally asymptotically stable. In the delay case, we present sufficient conditions for the global attractivity of $N^{*}(t)$. Our results imply that under the appropriate periodic impulsive perturbations, the impulsive delay equation preserves the original periodic property of the nonimpulsive delay equation. In particular, our work extends and improves some known results.

Copyright (c) 2006 Jian Song. This is an open access article distributed under the Creative Commons Attribution License, which permits unrestricted use, distribution, and reproduction in any medium, provided the original work is properly cited.

\section{Introduction}

The theory of impulsive differential equations has attracted the interest of many researchers in the past twenty years $[1,2,9-14,16]$ since they provide a natural description of several real processes subject to certain perturbations whose duration is negligible in comparison with the duration of the process. Such processes are often investigated in various fields of science and technology such as physics, population dynamics, ecology, biological systems, optimal control, and so forth. For details, see [1,9] and references therein. Recently, the corresponding theory for impulsive functional differential equations has been studied by several authors $[2,5,10-12,15]$.

The nonlinear delay differential equation

$$
N^{\prime}(t)=r N(t)\left(\frac{K-N(t-\tau)}{K+\lambda N(t-\tau)}\right)
$$

Hindawi Publishing Corporation

Discrete Dynamics in Nature and Society

Volume 2006, Article ID 31614, Pages 1-10

DOI 10.1155/DDNS/2006/31614 
where $m$ is a positive integer and that

$$
r(t), K(t), \lambda(t) \text { are positive periodic functions of periodic } \omega
$$

has been proposed by Smith (see [4]) for a "food-limited" population model. He demonstrated that the average growth rate is linear. It is not realistic (for the Daphnia populations). For more details of (1.1), one can refer to $[3,4,7]$.

Recently, taking into account the effects of a periodically varying environment, Huo and Li [6] considered the following delay "food-limited" population model:

$$
N^{\prime}(t)=r(t) N(t)\left(\frac{K(t)-N(t-m \omega)}{K(t)+\lambda(t) N(t-m \omega)}\right)
$$

and obtained sufficient conditions for existence and global attractivity of positive periodic of (1.3).

With the ideal of impulsive perturbation, we will study the existence of positive periodic solution of the following impulsive delay periodic "food-limited" population model:

$$
\begin{gathered}
N^{\prime}(t)=r(t) N(t)\left(\frac{K(t)-N(t-m \omega)}{K(t)+\lambda(t) N(t-m \omega)}\right), \quad \text { a.e. } t>0, t \neq t_{k} \\
N\left(t_{k}^{+}\right)=\left(1+b_{k}\right) N\left(t_{k}\right), \quad k=1,2, \ldots,
\end{gathered}
$$

where $m$ is a positive integer, $r(t), K(t), \lambda(t)$ are positive periodic functions of periodic $\omega>0$.

Next, we will consider (1.4). In the nondelay case $(m=0)$, we will show that (1.4) has a unique positive periodic solution $N^{*}(t)$, which is global asymptotically stable. In the delay case, we will establish sufficient conditions for the global attractivity of $N^{*}(t)$. Our results imply that under the appropriate periodic impulsive perturbations, the impulsive delay equation (1.4) preserves the original periodicity of the nonimpulsive delay differential equation. In particular, our work extends the results of Huo and $\mathrm{Li}$ [6] for the nonimpulsive delay population model.

For the system (1.4), we make the following assumptions:

$\left(\mathrm{A}_{1}\right) 0<t_{1}<t_{2}<\cdots$ are fixed impulsive points with $t_{k} \rightarrow+\infty$ as $k \rightarrow \infty$;

$\left(\mathrm{A}_{2}\right)\left\{b_{k}\right\}$ is a real sequence and $b_{k}>-1, k=1,2, \ldots$;

$\left(\mathrm{A}_{3}\right) r(t), K(t), \lambda(t)$ and $\prod_{0<t_{k}<t}\left(1+b_{k}\right)$ are periodic functions with period $\omega>0, m \geq 0$ is an integer.

Throughout this paper we always assume that a product equals unit if the number of factors is zero. Let

$$
\bar{f}=\frac{1}{m \omega} \int_{0}^{m \omega} f(t) d t, \quad f_{m}=\min _{t \in[0, \omega]} f(t), \quad f_{M}=\max _{t \in[0, \omega]} f(t),
$$

where $f$ is a periodic continuous positive function with period $\omega$. We will consider (1.4) with the initial condition

$$
N(t)=\varphi(t), \quad \text { for } t \in[-m \omega, 0], \varphi(t) \in \mathbb{C}([-m \omega, 0],[0, \infty)), \quad \varphi(0)>0
$$


Definition 1.1. A function $N \in([-m \omega,+\infty),(0,+\infty))$ is said to be a solution of (1.4) on $[-m \omega,+\infty)$ if

(i) $N(t)$ is absolutely continuous on each interval $\left(0, t_{1}\right]$ and $\left(t_{k}, t_{k+1}\right], k=1,2, \ldots$;

(ii) for any $t_{k}, k=1,2, \ldots, N\left(t_{k}^{+}\right)$and $N\left(t_{k}^{-}\right)$exist and $N\left(t_{k}^{-}\right)=N\left(t_{k}\right)$;

(iii) $N(t)$ satisfies the former equation of (1.4) for a.e. in $[0,+\infty) \backslash\left\{t_{k}\right\}$ and satisfies the latter equation for every $t=t_{k}, k=1,2, \ldots$.

Under the hypotheses $\left(A_{1}\right)-\left(A_{3}\right)$, we consider the nonimpulsive delay differential equation

$$
z^{\prime}(t)=r(t) z(t)\left(\frac{\rho(t)-z(t-m \omega)}{\rho(t)+\lambda(t) z(t-m \omega)}\right)
$$

with initial condition

$$
z(t)=\varphi(t), \quad \text { for } t \in[-m \omega, 0], \varphi(t) \in \mathbb{C}([-m \omega, 0],[0, \infty)), \quad \varphi(0)>0,
$$

where

$$
\rho(t)=\frac{K(t)}{\prod_{0 \leq t_{k}<t-m \omega}\left(1+b_{k}\right)}, \quad \text { for } t>0 .
$$

By a solution of (1.7) and (1.8) we mean an absolutely continuous function $z(t)$ defined on $[-m \omega,+\infty)$ satisfying (1.7) a.e. for $t \geq 0$ and $z(t)=\varphi(t)$ on $[-m \omega, 0]$.

The following lemma will be used in the proofs of our results. The proof is similar to that of [16, Theorem 1]. We will omit it here.

Lemma 1.2. Assume that $\left(A_{1}\right)-\left(A_{3}\right)$ hold. Then

(i) if $z(t)$ is a solution of (1.7) on $[-m \omega,+\infty)$, then $N(t)=\prod_{0<t_{k}<t}\left(1+b_{k}\right) z(t)$ is a solution of (1.4) on $[-m \omega,+\infty)$;

(ii) if $N(t)$ is a solution of (1.4) on $[-m \omega,+\infty)$, then $z(t)=\prod_{0<t_{k}<t}\left(1+b_{k}\right)^{-1} N(t)$ is a solution of (1.7) on $[-m \omega,+\infty)$.

It is easy to know that the solutions of (1.7) are defined on $[-m \omega,+\infty]$ and are positive on $[0, \infty)$.

\section{Results in the nondelay case}

In this section, we will study the periodic and asymptotic behavior of all solutions of (1.7) without delay, that is,

$$
z^{\prime}(t)=r(t) z(t)\left(\frac{\rho(t)-z(t)}{\rho(t)+\lambda(t) z(t)}\right)
$$

We will prove that there exists a unique positive periodic solution $z^{*}$ which is global asymptotically stable. 
Theorem 2.1. Assume that $\left(A_{1}\right)-\left(A_{3}\right)$ hold. Then

(a) there exists a unique $\omega$-periodic positive solution $\bar{z}(t)$ of (2.1),

(b) for every other positive solution $z(t)$ of (2.1),

$$
\lim _{t \rightarrow \infty}[z(t)-\bar{z}(t)]=0 .
$$

Proof. To prove (a), we define function

$$
f(z)=\frac{r \rho-r z}{\rho+\lambda z}, \quad z \in(0, \infty),
$$

where $r, \rho$, and $\lambda$ are positive constants. Clearly, $f(0)=r, f(\infty)=-r / \lambda$, and

$$
f^{\prime}(z)=-\frac{r \rho(1+\lambda)}{(\rho+\lambda z)^{2}}<0, \quad \forall z>0 .
$$

Thus, $f(z)=0$ has a unique positive root $z_{0} \in(0, \infty)$. Furthermore, $f(z)>0, z \in\left[0, z_{0}\right]$ and $f(z)<0, z \in\left[z_{0}, \infty\right)$.

Now, we define two functions

$$
f_{1}(z)=\frac{r_{m} \rho_{m}-r_{M} z}{\rho_{M}+\lambda_{M} z}, \quad f_{2}(z)=\frac{r_{M} \rho_{M}-r_{m} z}{\rho_{m}+\lambda_{m} z}
$$

Then from the above discussion, for $z>0, f_{1}(z)$ and $f_{2}(z)$ have zeros $z_{1}$ and $z_{2}$, respectively, that is, $f_{1}\left(z_{1}\right)=0$ and $f_{2}\left(z_{2}\right)=0$. Further, since

$$
\frac{r_{m} \rho_{m}-r_{M} z_{1}}{\rho_{M}+\lambda_{M} z_{1}}<\frac{r_{M} \rho_{M}-r_{m} z_{2}}{\rho_{m}+\lambda_{m} z_{2}}=0
$$

it is clear that $z_{2}>z_{1}$. Now, suppose $z(t)=z\left(t, 0, z_{0}\right)$ with $z_{0}>0$ is the unique solution of (2.1) through $\left(0, z_{0}\right)$. We claim that $z_{0} \in\left[z_{1}, z_{2}\right]$ implies that $z(t) \in\left[z_{1}, z_{2}\right]$ for all $t \geq 0$. Otherwise, let $t^{*}=\inf \left\{t>0 \mid z(t)>z_{2}\right\}$. Then, there exists $\bar{t} \geq t^{*}$ such that $z(\bar{t})>z_{2}$ and $z^{\prime}(\bar{t}) \geq 0$. However, then from (2.1) and the fact that $z(\bar{t})>z_{2}$, we conclude that for $\bar{t} \geq t^{*}$

$$
\begin{aligned}
z^{\prime}(\bar{t}) & =r(\bar{t}) z(\bar{t})\left[\frac{\rho(\bar{t})-z(\bar{t})}{\rho(\bar{t})+\lambda(\bar{t}) z(\bar{t})}\right] \\
& <z(\bar{t})\left[\frac{r_{M} \rho_{M}-r_{m} z_{2}}{\rho_{m}+\lambda_{m} z_{2}}\right]=0,
\end{aligned}
$$

which is a contradiction. By a similar argument, we can show that $z(t)>z_{1}$ for all $t \geq 0$. Hence, in particular, $z_{\omega}=z\left(\omega, 0, z_{0}\right) \in\left[z_{1}, z_{2}\right]$.

We define a mapping $F:\left[z_{1}, z_{2}\right] \rightarrow\left[z_{1}, z_{2}\right]$ as follows: for each $z_{0} \in\left[z_{1}, z_{2}\right], F\left(z_{0}\right)=z_{\omega}$. Since the solution $z\left(t, 0, z_{0}\right)$ depends continuously on the initial value $z_{0}$, it follows that $F$ is continuous and maps the interval $\left[z_{1}, z_{2}\right]$ into itself. By Brouwer fixed theorem, $F$ has a fixed point $\bar{z}_{0}$. In view of the periodicity of $r(t), \rho(t)$, and $\lambda(t)$, it follows that the unique solution $\bar{z}(t)=z\left(t, 0, \bar{z}_{0}\right)$ of $(2.1)$ through the initial point $\left(0, z_{0}\right)$ is a positive periodic solution of period $\omega$. The proof of (a) is complete. 
Now we will prove (b). Assume that $z(t)>\bar{z}(t)$ for $t$ sufficiently large (the proof when $z(t)<\bar{z}(t)$ is similar and will be omitted).

Set

$$
z(t)=\bar{z}(t) e^{w(t)}
$$

Then, $w(t)>0$ for $t$ sufficiently large, and satisfies

$$
w^{\prime}(t)+\frac{r(t) \rho(t)-r(t) \bar{z}(t)}{\rho(t)+\lambda(t) \bar{z}(t)}-\frac{r(t)\left(\rho(t)-\bar{z}(t) e^{w(t)}\right)}{\rho(t)+\lambda(t) \bar{z}(t) e^{w(t)}}=0,
$$

or

$$
w^{\prime}(t)+\frac{r(t) \rho(t) \bar{z}(t)\left(e^{w(t)}-1\right)(1+\lambda(t))}{(\rho(t)+\lambda(t) \bar{z}(t))\left(\rho(t)+\lambda(t) \bar{z}(t) e^{w(t)}\right)}=0 .
$$

However, since $e^{w(t)}-1>0$ for $t$ sufficiently large, it follows that

$$
w^{\prime}(t)=-\frac{r(t) \rho(t) \bar{z}(t)\left(e^{w(t)}-1\right)(1+\lambda(t))}{(\rho(t)+\lambda(t) \bar{z}(t))\left(\rho(t)+\lambda(t) \bar{z}(t) e^{w(t)}\right)}<0 .
$$

Thus, $w(t)$ is decreasing, and therefore $\lim _{t \rightarrow \infty} w(t)=\alpha \in[0, \infty)$. Now we will prove that $\alpha=0$. If $\alpha>0$, then there exists $\epsilon>0$ and $T_{\epsilon}>0$ such that $t \geq T_{\epsilon}, 0<\alpha-\epsilon<w(t)<\alpha+\epsilon$. However, from (2.11), we have

$$
w^{\prime}(t) \leq-\frac{r_{m} \rho_{m} \bar{z}_{m}\left(e^{\alpha-\epsilon}-1\right)\left(1+\lambda_{m}\right)}{\left(\rho_{M}+\lambda_{M} \bar{z}_{M}\right)\left(\rho_{M}+\lambda_{M} \bar{z}_{M} e^{\alpha+\epsilon}\right)}, \quad t \geq T_{\epsilon}
$$

But, now an integration of (2.12) from $T_{\epsilon}$ to $\infty$ immediately gives a contradiction. Hence, $\alpha=0$ and therefore $w(t)$ tends to zero as $t \rightarrow \infty$. Thus, we have

$$
\lim _{t \rightarrow \infty}[z(t)-\bar{z}(t)]=\lim _{t \rightarrow \infty} \bar{z}(t)\left(e^{w(t)}-1\right)=0 .
$$

The proof of (b) is completed.

Remark 2.2. From the proof of Theorem 2.1, it follows that the unique $\omega$-periodic positive solution $\bar{z}(t)$ of $(2.1)$ satisfies $z_{1} \leq \bar{z}(t) \leq z_{2}$. Thus, an interval for the location of $\bar{z}(t)$ is readily available.

\section{Results in the delay case}

In this section, we will consider the periodic delay differential equation (1.7). It is easy to see that the unique periodic positive solution $\bar{z}(t)$ of $(2.1)$ is also a periodic positive solution of (1.7). Conversely, if (1.7) and (1.8) have an $\omega$-periodic solution $\bar{z}(t)$, then such a solution is also a periodic solution of (2.1). Hence (1.7) has a unique $\omega$-periodic solution $\bar{z}(t)$.

In the following we first prove that every positive solution of (1.7) which does not oscillate about $\bar{z}(t)$ converges to $\bar{z}(t)$. Finally, we will establish sufficient conditions for $\bar{z}(t)$ to be a global attractor of all other positive solutions of (1.7). 
Theorem 3.1. Assume that $\left(A_{1}\right)-\left(A_{3}\right)$ hold. Let $z(t)$ be a positive solution of (1.7) which does not oscillate about $\bar{z}(t)$. Then

$$
\lim _{t \rightarrow \infty}[z(t)-\bar{z}(t)]=0
$$

Proof. The proof is similar to that of Theorem 2.1 and it will be omitted.

To show that $\bar{z}(t)$ is a global attractor of (1.7) we also need the following lemma.

Lemma 3.2. Assume that $\left(A_{1}\right)-\left(A_{3}\right)$ hold, and let $z(t)$ be a positive solution of (1.7) which oscillates about $\bar{z}(t)$. Then, there exists a $T$ such that for all $t \geq T$,

$$
Z_{1}=z_{1} \exp \overline{\left(\frac{r(t) \rho(t)-r(t) z_{2}}{\rho(t)+\lambda(t) z_{2}}\right)} m \omega \leq z(t) \leq z_{2} \exp m \omega \overline{(r(t))}=Z_{2} .
$$

Proof. First we will show the following inequality:

$$
z(t) \leq z_{2} \exp m \omega \overline{(r(t))}=Z_{2}
$$

Let $m \omega \leq t_{1}<t_{2}<\cdots<t_{l}<\cdots$ be a sequence of zeros $z(t)-\bar{z}(t)$ with $\lim _{t \rightarrow \infty} t_{l}=\infty$. Our strategy is to show that the upper bound holds in each interval $\left(t_{l}, t_{l+1}\right)$. For this, let $\zeta_{l} \in\left(t_{l}, t_{l+1}\right)$ be a point where $z(t)$ attends its maximum in $\left(t_{l}, t_{l+1}\right)$. Then, it suffices to show that

$$
z\left(\zeta_{l}\right) \leq z_{2} \exp m \omega \overline{(r(t))}=Z_{2}
$$

We can assume that there exists a $\zeta_{l}$ where $z\left(\zeta_{l}\right)>z_{2}$, otherwise there is nothing to prove. Since $z^{\prime}\left(\zeta_{l}\right)=0$, it follows that

$$
0=z^{\prime}\left(\zeta_{l}\right)<z\left(\zeta_{l}\right)\left(\frac{r_{M} \rho_{M}-r_{m} z\left(\zeta_{l}-m \omega\right)}{\rho_{m}+\lambda_{m} z\left(\zeta_{l}-m \omega\right)}\right)
$$

and hence

$$
\frac{r_{M} \rho_{M}-r_{m} z\left(\zeta_{l}-m \omega\right)}{\rho_{m}+\lambda_{m} z\left(\zeta_{l}-m \omega\right)}>0
$$

Thus, if $z(t)$ attends its maximum at $\zeta_{l}$, then it follows (cf. see the proof of Theorem 2.1) that $z\left(\zeta_{l}-m \omega\right)<z_{2}$. Since $z\left(\zeta_{l}\right)>z_{2}$ and $z\left(\zeta_{l}-m \omega\right)<z_{2}$, we can let $\bar{\zeta}_{l}$ be the first zero of $z(t)-z_{2}$ in $\left(\zeta_{l}-m \omega, \zeta_{l}\right)$, that is, $z\left(\bar{\zeta}_{l}\right)=z_{2}$. Integrating (1.7) from $\bar{\zeta}_{l}$ to $\zeta_{l}$, we get

$$
\begin{aligned}
\ln \frac{z\left(\zeta_{l}\right)}{z\left(\bar{\zeta}_{l}\right)} & =\int_{\bar{\zeta}_{l}}^{\zeta_{l}}\left(\frac{r(t) \rho(t)-r(t) z(t-m \omega)}{\rho(t)+\lambda(t) z(t-m \omega)}\right) d t \\
& <\int_{\bar{\zeta}_{l}}^{\zeta_{l}} \frac{\rho(t) r(t)}{\rho(t)} d t<\overline{(r(t))} m \omega,
\end{aligned}
$$

which immediately gives (3.4). 
Now, we will show the following inequality for $t \geq T_{1}+m \omega$ :

$$
Z_{1}=z_{1} \exp \left(\int_{0}^{m \omega}\left(\frac{r(t) \rho(t)-r(t) z_{2}}{\rho(t)+\lambda(t) z_{2}}\right) d t\right) \leq z(t) .
$$

For this, following as above let $\mu_{l} \in\left(t_{l}, t_{l+1}\right)$ be a point where $z(t)$ attends its minimum in $\left(t_{l}, t_{l+1}\right)$. Then, it suffices to show that

$$
Z_{1}=z_{1} \exp \left(\int_{0}^{m \omega}\left(\frac{r(t) \rho(t)-r(t) z_{2}}{\rho(t)+\lambda(t) z_{2}}\right) d t\right) \leq z\left(\mu_{l}\right) .
$$

Since $Z_{2}>z_{2}>z_{1}$, it follows that

$$
\frac{r_{m} \rho_{m}-r_{M} z_{2}}{\rho_{M}+\lambda_{M} z_{2}}<0
$$

Thus, $Z_{1}<z_{1}$. Now, assume that there exists a $\mu_{l} \geq T_{1}+m \omega$ where $z\left(\mu_{l}\right)<z_{1}$, otherwise there is nothing to prove. Since $z^{\prime}\left(\mu_{l}\right)=0$, we have

$$
0=z^{\prime}\left(\mu_{l}\right)>z\left(\mu_{l}\right)\left(\frac{r_{m} \rho_{m}-r_{M} z\left(\mu_{l}-m \omega\right)}{\rho_{M}+\lambda_{M} z\left(\mu_{l}-m \omega\right)}\right)
$$

and hence

$$
\frac{r_{m} \rho_{m}-r_{M} z\left(\mu_{l}-m \omega\right)}{\rho_{M}+\lambda_{M} z\left(\mu_{l}-m \omega\right)}<0 .
$$

Thus, it is necessary that $z\left(\mu_{l}-m \omega\right)>z_{1}$. Hence, there exists a $\bar{\mu}_{l} \in\left(\mu_{l}-m \omega, \mu_{l}\right)$ where $z\left(\bar{\mu}_{l}\right)=z_{1}$. Integrating (1.7) from $\bar{\mu}_{l}$ to $\mu_{l}$, and using $z(t) \leq z_{2}$ and (3.10), we get

$$
\begin{aligned}
\ln \frac{z\left(\mu_{l}\right)}{z\left(\bar{\mu}_{l}\right)} & =\int_{\bar{\mu}_{l}}^{\mu_{l}}\left(\frac{r(t) \rho(t)-r(t) z(t-m \omega)}{\rho(t)+\lambda(t) z(t-m \omega)}\right) d t \\
& >\int_{\bar{\mu}_{l}}^{\mu_{l}}\left(\frac{r(t) \rho(t)-r(t) z_{2}}{\rho(t)+\lambda(t) z_{2}}\right) d t \\
& >m \omega \overline{\left(\frac{r(t) \rho(t)-r(t) z_{2}}{\rho(t)+\lambda(t) z_{2}}\right)},
\end{aligned}
$$

which immediately leads to (3.9).

The following result provides sufficient conditions for the global attractivity of $\bar{z}(t)$.

Theorem 3.3. Assume that $\left(A_{1}\right)-\left(A_{3}\right)$ hold, and

$$
\lim _{t \rightarrow \infty} \int_{t-m \omega}^{t}\left((1+\lambda(s)) \frac{K(s) r(s) Y_{2}\left(K(s)+\lambda(s) Y_{1}\right)}{\left(K(s)+\lambda(s) Y_{1}\right) K(s)^{2}}\right) d s<\frac{\pi}{2},
$$

where $Z_{1}$ and $Z_{2}$ are as in (3.2). Then,

$$
\lim _{t \rightarrow \infty}[z(t)-\bar{z}(t)]=0 .
$$


Proof. In the nondelay case we have established (3.15) in Theorem 2.1(b), and for the positive solutions of (1.7) which are nonoscillatory about $\bar{z}(t)$ we have shown (3.15) in Theorem 3.1. Thus, it remains to prove (3.15) for the positive solutions of (1.7) which oscillate about $\bar{z}(t)$. If $z(t)$ is an arbitrary positive solution of (1.7), then from the transformation (2.8), (1.7) reduces to

$$
w^{\prime}(t)+(\lambda(t)+1) \frac{K(t) r(t) \bar{z}(t)\left(e^{w(t-m \omega)}-1\right)}{(K(t)+\lambda(t) \bar{z}(t-m \omega))\left(K(t)+\lambda(t) \bar{z}(t-m \omega) e^{w(t-m \omega)}\right)}=0 .
$$

Let

$$
G(t, u)=(\lambda(t)+1) \frac{K(t) r(t) \bar{z}(t)\left(e^{u}-1\right)}{(K(t)+\lambda(t) \bar{z}(t-m \omega))\left(K(t)+\lambda(t) \bar{z}(t-m \omega) e^{u}\right)} .
$$

Then, we have

$$
\frac{\partial G(t, u)}{\partial u}=(\lambda(t)+1) \frac{K(t) r(t) \bar{z}(t)\left(e^{u}-1\right)(K(t)+\lambda(t) \bar{z}(t-m \omega)) e^{u}}{(K(t)+\lambda(t) \bar{z}(t-m \omega))\left(K(t)+\lambda(t) \bar{z}(t-m \omega) e^{u}\right)^{2}} .
$$

Equation (3.16) is the same as

$$
w^{\prime}(t)+G(t, w(t-m \omega))-G(t, 0)=0 \text {. }
$$

Clearly, by the mean value theorem (3.19) can be written as

$$
w^{\prime}(t)+F(t) w(t-m \omega)=0
$$

where

$$
\begin{aligned}
F(t) & =\left.\frac{\partial G(t, u)}{\partial u}\right|_{u=\xi(t)} \\
& =(\lambda(t)+1) \frac{K(t) r(t) \bar{z}(t)(K(t)+\lambda(t) \bar{z}(t-m \omega)) e^{\xi(t)}}{(K(t)+\lambda(t) \bar{z}(t-m \omega))\left(K(t)+\lambda(t) \bar{z}(t-m \omega) e^{\xi(t)}\right)^{2}}
\end{aligned}
$$

and $\xi(t)$ lies between 0 and $w(t-m \omega)$, that is, $\min \{0, w(t-m \omega)\}<\xi(t)<\max \{0, w(t-$ $m \omega)\}$. Then by Lemma 3.2, we obtain

$$
\begin{gathered}
F(t) \geq(\lambda(t)+1) \frac{K(t) r(t) Z_{1}\left(K(t)+\lambda(t) Z_{1}\right)}{\left(K(t)+\lambda(t) Z_{2}\right)\left(K(t)+\lambda(t)\left(Z_{2} / Z_{1}\right) Z_{2}\right)^{2}}, \\
F(t) \leq(\lambda(t)+1) \frac{K(t) r(t) Z_{2}\left(K(t)+\lambda(t) Z_{1}\right)}{\left(K(t)+\lambda(t) Z_{1}\right)(K(t))^{2}} .
\end{gathered}
$$

Thus, in view of (3.14), we find

$$
\lim _{t \rightarrow \infty} \int_{t-m \omega}^{t} F(s) d s \leq \lim _{t \rightarrow \infty} \int_{t-m \omega}^{t}\left((1+\lambda(s)) \frac{K(s) r(s) Z_{2}\left(K(s)+\lambda(s) Z_{1}\right)}{\left(K(s)+\lambda(s) Z_{1}\right) K(s)^{2}}\right) d s<\frac{\pi}{2} .
$$


But, now by a known result in [8], every solution of (3.20) satisfies $\lim _{t \rightarrow \infty} w(t)=0$, and hence $\lim _{t \rightarrow \infty}[z(t)-\bar{z}(t)]=0$.

Remark 3.4. Our results in this paper indicate that under the appropriate periodic impulsive perturbations, the impulsive delay model (1.4) preserves the original periodicity of the nonimpulsive delay model (1.7).

Remark 3.5. Next, we consider the following nonimpulsive delay periodic "food-limited" population model:

$$
x^{\prime}(t)+\frac{\alpha V(t) x(t) x^{n}(t-m \omega)}{\theta^{n}+x^{n}(t-m \omega)}=\lambda(t)
$$

By employing Theorem 3.3, we obtained sufficient conditions of global attractivity of positive periodic solution of (3.24). The conditions which we have obtained are given in terms of the averages of the related parameters over an interval of the common period. So our results generalized the main results in [6] which are given in terms of supremum and infimum of the parameters.

\section{References}

[1] D. D. Baĭnov and P. S. Simeonov, Systems with Impulse Effect. Stability, Theory and Applications, Ellis Horwood Series: Mathematics and Its Applications, Ellis Horwood, Chichester, 1989.

[2] G. Ballinger and X. Liu, Existence and uniqueness results for impulsive delay differential equations, Dynamics of Continuous, Discrete and Impulsive Systems 5 (1999), no. 1-4, 579-591.

[3] K. Gopalsamy, Stability and Oscillations in Delay Differential Equations of Population Dynamics, Mathematics and Its Applications, vol. 74, Kluwer Academic, Dordrecht, 1992.

[4] K. Gopalsamy, M. R. S. Kulenović, and G. Ladas, Oscillations and global attractivity in respiratory dynamics, Dynamics and Stability of Systems 4 (1989), no. 2, 131-139.

[5] K. Gopalsamy and B. G. Zhang, On delay differential equations with impulses, Journal of Mathematical Analysis and Applications 139 (1989), no. 1, 110-122.

[6] H. F. Huo and W. T. Li, Global attractivity and oscillation in a periodic "food-limited" population model with delay, Acta Mathematica Scientia 25 (2005), no. 2, 158-165.

[7] Y. Kuang, Delay Differential Equations with Applications in Population Dynamics, Mathematics in Science and Engineering, vol. 191, Academic Press, Massachusetts, 1993.

[8] G. Ladas, Y. G. Sficas, and I. P. Stavroulakis, Asymptotic behavior of solutions of retarded differential equations, Proceedings of the American Mathematical Society 88 (1983), no. 2, 247-253.

[9] V. Lakshmikantham, D. D. Bainnov, and P. S. Simeonov, Theory of Impulsive Differential Equations, Series in Modern Applied Mathematics, vol. 6, World Scientific, New Jersey, 1989.

[10] X. Liu and G. Ballinger, Uniform asymptotic stability of impulsive delay differential equations, Computers \& Mathematics with Applications 41 (2001), no. 7-8, 903-915.

[11] Existence and continuability of solutions for differential equations with delays and statedependent impulses, Nonlinear Analysis. Theory, Methods \& Applications 51 (2002), no. 4, 633647.

[12] B__ Boundedness for impulsive delay differential equations and applications to population growth models, Nonlinear Analysis. Theory, Methods \& Applications 53 (2003), no. 7-8, 10411062.

[13] S. G. Pandit, On the stability of impulsively perturbed differential systems, Bulletin of the Australian Mathematical Society 17 (1977), no. 3, 423-432. 


\section{An impulsive population model}

[14] M. G. Roberts and R. R. Kao, The dynamics of an infectious disease in a population with birth pulses, Mathematical Biosciences 149 (1998), no. 1, 23-36.

[15] J. H. Shen, Global existence and uniqueness, oscillation, and nonoscillation of impulsive delay differential equations, Acta Mathematica Sinica 40 (1997), no. 1, 53-59 (Chinese).

[16] J. Yan, Existence and global attractivity of positive periodic solution for an impulsive LasotaWazewska model, Journal of Mathematical Analysis and Applications 279 (2003), no. 1, 111120.

Jian Song: College of Network Education, Lanzhou University of Technology, Lanzhou,

Gansu 730050, China

E-mail address: songj@lut.cn 


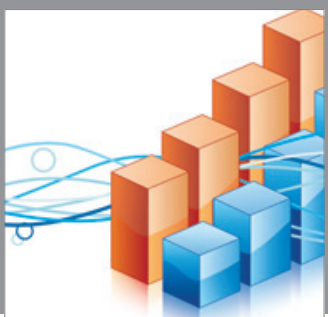

Advances in

Operations Research

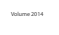

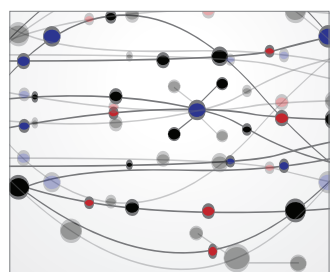

\section{The Scientific} World Journal
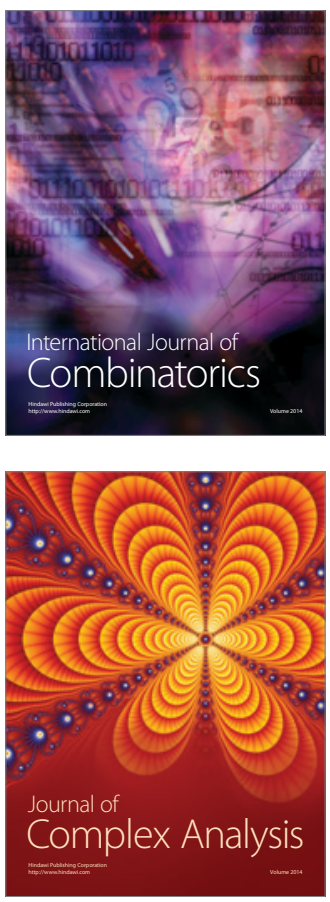

International Journal of

Mathematics and

Mathematical

Sciences
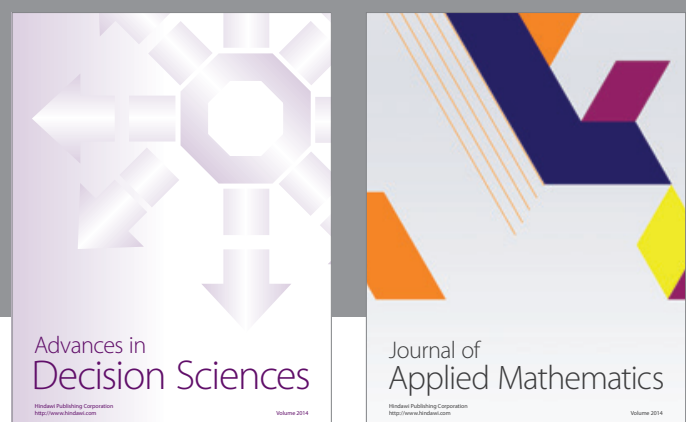

Journal of

Applied Mathematics
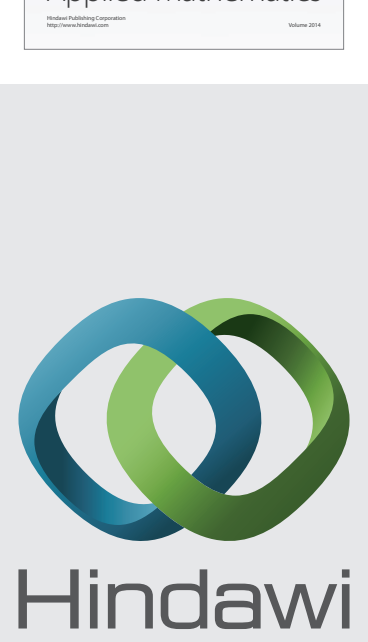

Submit your manuscripts at http://www.hindawi.com
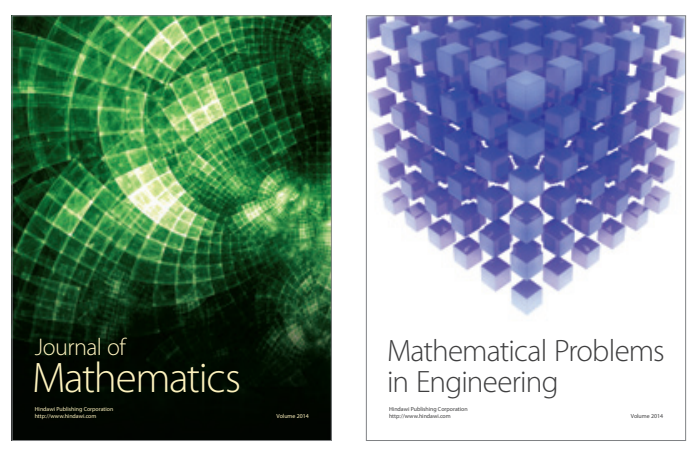

Mathematical Problems in Engineering
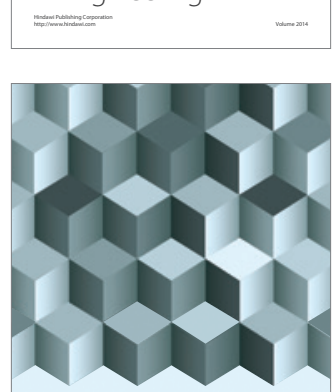

Journal of

Function Spaces
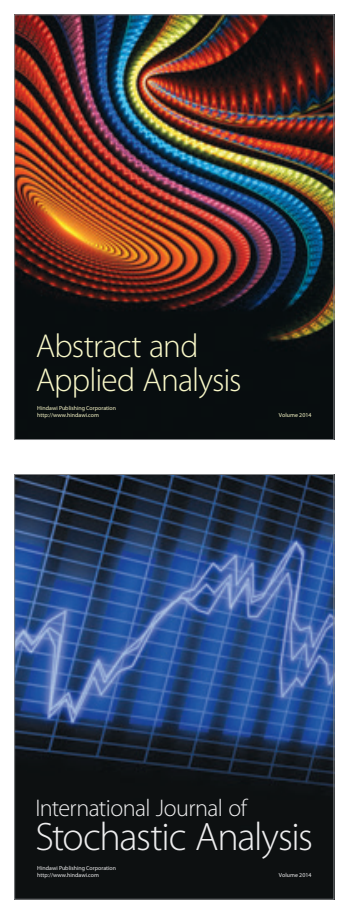

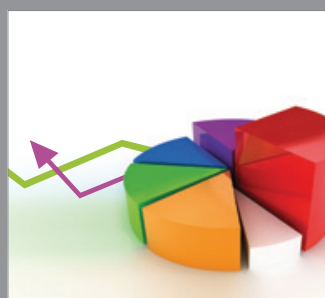

ournal of

Probability and Statistics

Promensencen
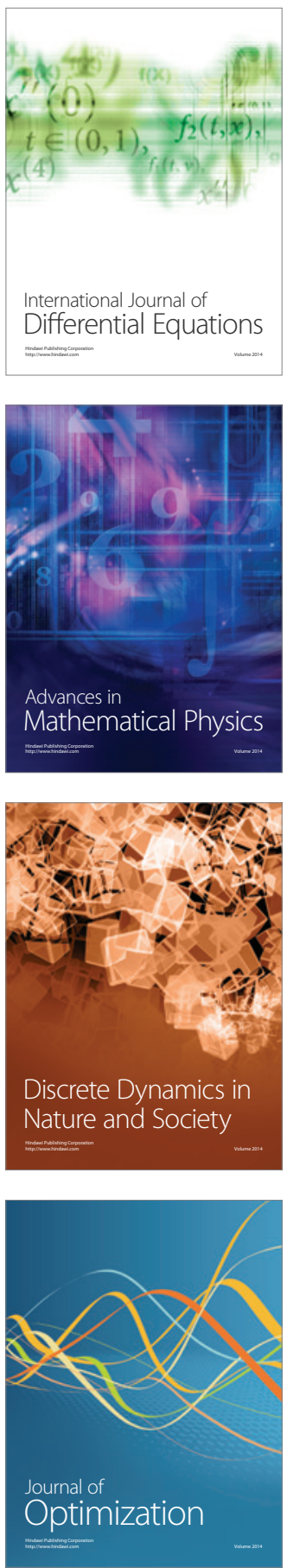